\title{
Kramers-Kronig relations for plasma-like permittivities and the Casimir force
}

\author{
G L Klimchitskaya ${ }^{1}$, U Mohideen ${ }^{2}$ and V M Mostepanenko ${ }^{3}$ \\ ${ }^{1}$ North-West Technical University, Millionnaya St. 5, St.Petersburg, Russia \\ 2 Department of Physics and Astronomy, University of California, Riverside, CA \\ 92521, USA \\ ${ }^{3}$ Noncommercial Partnership "Scientific Instruments", Tverskaya St. 11, Moscow, \\ Russia.
}

\begin{abstract}
The Kramers-Kronig relations are derived for the permittivity of the usual plasma model which neglects dissipation and of a generalized model which takes into account the interband transitions. The generalized plasma model is shown to be consistent with all precision experiments on the measurement of the Casimir force.

PACS numbers: 05.30.-d, 77.22.Ch, 12.20.Ds
\end{abstract}

\section{Introduction}

It is common knowledge that the Kramers-Kronig relations connect real and imaginary parts of an analytic function describing some causal physical process. In statistical physics and electrodynamics any material susceptibility satisfies the Kramers-Kronig relations [1, 2]. Specifically, they are used to calculate the real part of the dielectric permittivity, $\varepsilon^{\prime}(\omega)$, along the real frequency axis and the dielectric permittivity, $\varepsilon(i \xi)$, along the imaginary frequency axis [2, 3]. Both are expressed through the imaginary part of the permittivity, $\varepsilon^{\prime \prime}(\omega)$, at all real frequencies.

In the last few years the Kramers-Kronig relations have been repeatedly used to calculate the thermal Casimir force in the framework of the Lifshitz theory (see, e.g., reviews [4, 5] and recent proceedings [6]). The Casimir force [7] acts between neutral material bodies and originates from the zero-point oscillations of the electromagnetic field. The Lifshitz theory allows one to express the Casimir force at a temperature $T$ in terms of $\varepsilon(i \xi)$ of the body materials at discrete Matsubara frequencies $\xi_{l}=2 \pi k_{B} T l / \hbar$, where $k_{B}$ is the Boltzmann constant and $l=0,1,2, \ldots$ The tabulated optical data for the complex index of refraction and hence for $\varepsilon^{\prime \prime}(\omega)$ are, however, available only in some restricted frequency regions [8]. Therefore the direct calculation of $\varepsilon(i \xi)$ using the Kramers-Kronig relations is not possible and different approaches to find $\varepsilon(i \xi)$ have been proposed.

In the first approach [9, 10, the quantity $\varepsilon^{\prime \prime}(\omega)$ obtained from the tabulated optical data is extrapolated using the imaginary part of the Drude dielectric function to all lower 
frequencies including zero frequency. Then $\varepsilon\left(i \xi_{l}\right)$ at all $l \geq 0$ is found from the KramersKronig relations and is substituted into the Lifshitz formula at nonzero temperature. From the theoretical point of view this approach may seem straightforward, but it leads to a violation of the Nernst heat theorem for perfect crystal lattices with no impurities [11] and to contradiction with the experiment measuring the Casimir force at separations from 160 to $750 \mathrm{~nm}$ [12]. The second approach [12, 13] is based on the concept of the Leontovich surface impedance [2]. This approach leads to practically the same contributions to the Casimir force, as the first approach, at all Matsubara frequencies with $l \geq 1$. The contribution of the zero-frequency term is, however, different and fixed by the impedance used. As a consequence the second approach is not applicable at separations below the plasma wavelength (equal to $137 \mathrm{~nm}$ for $\mathrm{Au}$ ) where the Leontovich impedance boundary condition becomes invalid. The third approach [14, 15] does not use the tabulated optical data but employs the dielectric permittivity of the free electron plasma model at all frequencies. Both the second and the third approaches are in agreement with thermodynamics. They are also consistent with the experiment [12] performed at separations above the plasma wavelength. However, both the second and third approaches cannot be applied in the experiment [16, 17] where measurements start at short separations of $60 \mathrm{~nm}$. This experiment although performed at $T=300 \mathrm{~K}$ was found to be consistent with the Lifshitz theory at zero temperature. (The comparative analysis of all approaches is contained in [18-20].) Note that the third, plasma model approach, may seem to be in disagreement with the Kramers-Kronig relations because the dielectric permittivity of the plasma model is entirely real. In this connection the plasma model approach has been criticized [20] for the complete neglect of dissipation. Thus at the moment none of the theoretical approaches to the thermal Casimir force is consistent with all the available experimental information.

In the present paper we derive the generalized Kramers-Kronig relations for the permittivities of the free electron plasma and a plasma-like model which incorporates dissipation due to interband transitions. We demonstrate that the permittivity of the plasma model (as any function analytic in the upper half-plane) satisfies the KramersKronig relations if the contribution from the pole of the second order at zero frequency is correctly taken into account. Then we compare theoretical computations of the thermal Casimir force using the free-electron plasma model and the generalized plasma model incorporating interband transitions with the zero-temperature Casimir force calculated using the tabulated optical data. We demonstrate that the theoretical results using the generalized plasma model are in good agreement with experiment. Thus currently it is the only model for the thermal Casimir force which is consistent with all measurements performed to date. We conclude with a discussion of different types of dissipation processes and their role in the theoretical description of the Casimir force. 


\section{Kramers-Kronig relations for plasma and plasma-like models}

We consider the generalized plasma-like dielectric permittivity of the form [21]

$$
\varepsilon(\omega)-1=A(\omega)-\frac{\omega_{p}^{2}}{\omega^{2}}
$$

where $\omega_{p}$ is the plasma frequency and the oscillator term

$$
A(\omega)=\sum_{j=1}^{K} \frac{f_{j}}{\omega_{j}^{2}-\omega^{2}-i g_{j} \omega}
$$

takes into account the interband transitions of core electrons. Here $\omega_{j} \neq 0$ are the resonant frequencies of the core electrons, $g_{j}$ are the respective relaxation frequencies, $f_{j}$ are the oscillator strengths, and $K$ is the number of oscillators. The dielectric permittivity (11), (2) was used in Sec. 7.5(D) of [21] for the description of a metal at frequencies much larger than the Drude relaxation frequency. The term $-\omega_{p}^{2} / \omega^{2}$ in Eq. (1) describes the free conduction electrons and leads to a purely imaginary current. This contribution to $\varepsilon(\omega)$ is entirely real and does not include dissipation. It must be emphasized that the oscillator term (2) does not include the oscillator with zero resonant frequency $\omega_{0}=0$. Thus it does not describe conduction electrons but only the core electrons. If the core electrons were excluded from our consideration then $f_{j}=0$, $A(\omega)=0$, and the dielectric permittivity (11) leads to the usual plasma model. Note that for the purpose of the computations below we follow the notations from [22] (Level 2, D) for the parameters of the interband oscillators. Because of this we have replaced the relaxation parameter $\Gamma_{j}$ in [21] for $g_{j}$ and the oscillator strengths $4 \pi N e^{2} f_{j} / m$, where $N$ is the number of molecules per unit volume, as in [21], for $f_{j}$. Here we also use $1+A(\omega)$ in place of $\varepsilon_{b}(\omega)$ [21]. Equations (11) and (2) incorporate dissipation due to interband transitions but do not include processes of electron scattering on phonons, impurities, grain boundaries, surfaces and other electrons. Below we investigate the mathematical properties of Eqs. (1), (2) for the complete frequency range from zero to infinity. The physical justification for the choice of $\varepsilon(\omega)$ in Eq. (11) is discussed in Sec. 4.

The characteristic feature of the dielectric permittivity (1) is the second order pole at zero frequency. Let us demonstrate that (1) satisfies the Kramers-Kronig relations in

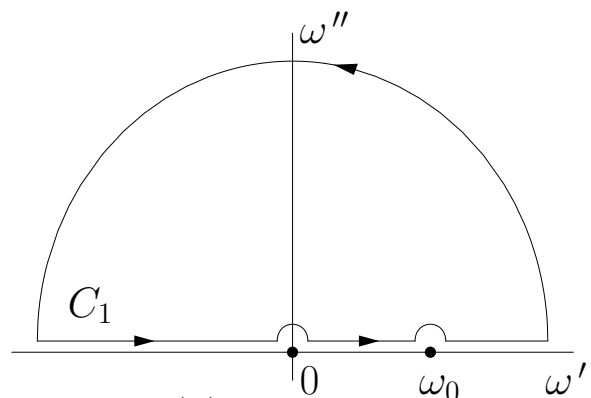

$(a)$

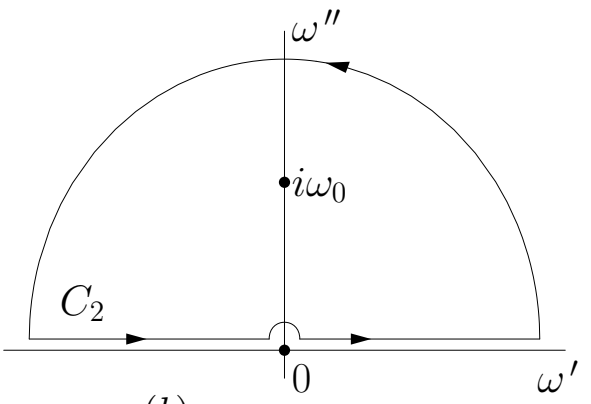

$(b)$

Figure 1. The integration contours ( $a$ ) in Eq. (3) and (b) in Eq. (11) consisting of the real frequency axis and the semicircle of infinitely large radius. 
both cases $A(\omega)=0$ and $A(\omega) \neq 0$. For this purpose we consider the integral

$$
\int_{C_{1}} \frac{\varepsilon(\omega)-1}{\omega-\omega_{0}} d \omega=0
$$

where $\omega_{0}$ is real and the contour $C_{1}$ is presented in Fig. 1(a). Inside $C_{1}$ the function under the integral is analytic and thus the equality (3) follows from the Cauchy theorem. At infinity $\varepsilon(\omega) \rightarrow 1$ and the function $[\varepsilon(\omega)-1] /\left(\omega-\omega_{0}\right)$ therefore tends to zero more rapidly than $1 / \omega$. Because of this the integral along the semicircle of infinite radius is zero. We pass around the points 0 and $\omega_{0}$ along the semicircles $C_{\rho}$ and $C_{\delta}$ with radii $\rho$ and $\delta$, respectively. It is easily seen that

$$
\int_{C_{\delta}} \frac{\varepsilon(\omega)-1}{\omega-\omega_{0}} d \omega=-\left.\pi i \operatorname{Res} \frac{\varepsilon(\omega)-1}{\omega-\omega_{0}}\right|_{\omega=\omega_{0}}=-\pi i\left[\varepsilon\left(\omega_{0}\right)-1\right] .
$$

The similar integral around the point 0 is more involved. Using Eq. (1) we represent it as a sum of the integral

$$
\int_{C_{\rho}} \frac{A(\omega)}{\omega-\omega_{0}} d \omega=-\frac{2 A(0)}{\omega_{0}} \rho,
$$

which vanishes when $\rho \rightarrow 0$, and

$$
-\omega_{p}^{2} \int_{C_{\rho}} \frac{d \omega}{\omega^{2}\left(\omega-\omega_{0}\right)} \equiv \frac{\omega_{p}^{2}}{\omega_{0}^{2}} \int_{C_{\rho}}\left[\frac{\omega_{0}}{\omega^{2}}-\frac{1}{\omega-\omega_{0}}+\frac{1}{\omega}\right] d \omega .
$$

Direct integration along the semicircle $C_{\rho}$ results in

$$
\begin{aligned}
& \int_{C_{\rho}} \frac{d \omega}{\omega-\omega_{0}}=-\frac{2}{\omega_{0}} \rho, \quad \int_{C_{\rho}} \frac{d \omega}{\omega}=-\pi i, \\
& \int_{C_{\rho}} \frac{d \omega}{\omega^{2}}=-\frac{2}{\rho}=\omega_{0} P \int_{-\infty}^{\infty} \frac{d \omega}{\omega^{2}\left(\omega-\omega_{0}\right)},
\end{aligned}
$$

where the integral is taken as a principal value. [Note that the last integral cannot be evaluated as in Eq. (4) because $\left.\operatorname{Res}\left(1 / \omega^{2}\right)\right|_{\omega=0}=0$ and both integrals around the upper and lower semicircles are divergent and opposite in sign.]

Substituting Eqs. (41)-(7) in Eq. (3) we arrive at

$$
-\frac{i \pi \omega_{p}^{2}}{\omega_{0}^{2}}-i \pi\left[\varepsilon\left(\omega_{0}\right)-1\right]+P \int_{-\infty}^{\infty} \frac{d \omega}{\omega-\omega_{0}}\left[\varepsilon(\omega)-1+\frac{\omega_{p}^{2}}{\omega^{2}}\right]=0 .
$$

Now we replace the integration variable $\omega$ by $\xi, \omega_{0}$ by $\omega$, and represent the function $\varepsilon(\omega)$ in the form of $\varepsilon(\omega)=\varepsilon^{\prime}(\omega)+i \varepsilon^{\prime \prime}(\omega)$. Taking into account that

$$
P \int_{-\infty}^{\infty} \frac{d \omega}{\omega-\omega_{0}}=0
$$

and separating the real and imaginary parts in Eq. (8), we obtain the generalized Kramers-Kronig relations

$$
\varepsilon^{\prime}(\omega)=1+\frac{1}{\pi} P \int_{-\infty}^{\infty} \frac{\varepsilon^{\prime \prime}(\xi)}{\xi-\omega} d \xi-\frac{\omega_{p}^{2}}{\omega^{2}}, \quad \varepsilon^{\prime \prime}(\omega)=-\frac{1}{\pi} P \int_{-\infty}^{\infty} \frac{\varepsilon^{\prime}(\xi)+\frac{\omega_{p}^{2}}{\xi^{2}}}{\xi-\omega} d \xi .(10)
$$

Note that the standard relations [2] obtained for permittivities with no pole at $\omega=0$ do not contain terms $\omega_{p}^{2} / \xi^{2}$ on the right-hand sides of Eqs. (10). 
The dielectric permittivity along the imaginary frequency axis can be determined through the use of the integral

$$
\int_{C_{2}} \frac{\omega[\varepsilon(\omega)-1]}{\omega^{2}+\omega_{0}^{2}} d \omega=\pi i\left[\varepsilon\left(i \omega_{0}\right)-1\right]
$$

along the contour $C_{2}$ in Fig. 1(b). By integrating over $C_{2}$ we get

$$
i \pi \frac{\omega_{p}^{2}}{\omega_{0}^{2}}+P \int_{-\infty}^{\infty} \frac{\omega[\varepsilon(\omega)-1]}{\omega^{2}+\omega_{0}^{2}} d \omega=i \pi\left[\varepsilon\left(i \omega_{0}\right)-1\right] .
$$

Now we make the same replacement of variables as above, separate the real and imaginary parts of $\varepsilon(\omega)$ under the integral and use the identities

$$
P \int_{-\infty}^{\infty} \frac{\xi d \xi}{\xi^{2}+\omega^{2}}=0, \quad P \int_{-\infty}^{\infty} \frac{\xi \varepsilon^{\prime}(\xi)}{\xi^{2}+\omega^{2}} d \xi=0
$$

The result is

$$
\varepsilon(i \omega)-1=\frac{1}{\pi} P \int_{-\infty}^{\infty} \frac{\xi \varepsilon^{\prime \prime}(\xi)}{\xi^{2}+\omega^{2}} d \xi+\frac{\omega_{p}^{2}}{\omega^{2}}
$$

For the usual plasma model $\varepsilon^{\prime \prime}(\omega)=0, \varepsilon^{\prime}(\omega)=1-\omega_{p}^{2} / \omega^{2}, \varepsilon(i \omega)=1+\omega_{p}^{2} / \omega^{2}$, the generalized Kramers-Kronig relations (10), (14) are satisfied with the use of Eq. (91). On the contrary the same plasma model violates the standard Kramers-Kronig relations. Note that sometimes [23] the plasma model is ascribed a nonzero imaginary part

$$
\varepsilon^{\prime \prime}(\omega)=-\frac{\omega_{p}^{2}}{\omega} \lim _{g \rightarrow 0} \frac{1}{\omega+i g}=\frac{\omega_{p}^{2}}{\omega} \pi \delta(\omega),
$$

which is obtained from the Drude dielectric function in the limit of zero relaxation parameter. This makes it possible to formally satisfy the standard dispersion relation for the dielectric permittivity along the imaginary frequency axis [2] given by Eq. (14) without the $\omega_{p}^{2} / \omega^{2}$ term. However the other two standard Kramers-Kronig relations with $\varepsilon^{\prime \prime}(\omega)$, as given by Eq. (15), become meaningless. Thus the permittivity of the collisionless free electron gas is entirely real.

It is easily seen that the plasma-like dielectric permittivity (11), (2) satisfies the generalized Kramers-Kronig relations (10) and (14). This can be verified by direct substitution. For example, the substitution of Eqs. (11) and (2) in the first equation of (10) leads to

$$
\begin{aligned}
\sum_{j=1}^{K} & \frac{f_{j}\left(\omega_{j}^{2}-\omega^{2}\right)}{\left(\omega_{j}^{2}-\omega^{2}\right)^{2}+g_{j}^{2} \omega^{2}}=\frac{1}{\pi} \sum_{j=1}^{K} f_{j} g_{j} P \int_{-\infty}^{\infty} \frac{\xi d \xi}{(\xi-\omega)\left[\left(\omega_{j}^{2}-\xi^{2}\right)^{2}+g_{j}^{2} \xi^{2}\right]} \\
= & \frac{1}{\pi} \sum_{j=1}^{K} \frac{f_{j} g_{j}}{\left(\omega_{j}^{2}-\omega^{2}\right)^{2}+g_{j}^{2} \omega^{2}} \\
& \times\left[\omega_{j} \int_{-\infty}^{\infty} \frac{d y}{y^{4}-2 \beta_{j} y^{2}+1}-\frac{\omega^{2}}{\omega_{j}} \int_{-\infty}^{\infty} \frac{y^{2} d y}{y^{4}-2 \beta_{j} y^{2}+1}\right]
\end{aligned}
$$

where $\beta_{j} \equiv 1-g_{j}^{2} /\left(2 \omega_{j}^{2}\right)$. When the following:

$$
\int_{-\infty}^{\infty} \frac{d y}{y^{4}-2 \beta_{j} y^{2}+1}=\int_{-\infty}^{\infty} \frac{y^{2} d y}{y^{4}-2 \beta_{j} y^{2}+1}=\frac{\pi}{\sqrt{2\left(1-\beta_{j}\right)}}
$$


is taken into account Eq. (16) is satisfied. The second equation in (10) and Eq. (14) can be verified in a similar way.

\section{Calculation of the Casimir force using the generalized plasma model and comparison with experiment}

We have calculated the thermal Casimir force $F^{\text {th }}$ acting between Au coated test bodies in the most precise short-separation experiment [16] (a sphere of $R=95.65 \mu \mathrm{m}$ radius and a plate) by substituting the generalized plasma dielectric function (11), (2) into the Lifshitz formula. The Lifshitz formula for the force between a sphere and a plate was obtained by means of the proximity force approximation (PFA) as $2 \pi R \mathcal{F}$ where $\mathcal{F}$ is the free energy in the configuration of two parallel plates (see [5, 17] for details). Note that recently the high accuracy of PFA was confirmed using the path-integral approach [24, 25] and worldline numerics [26]. The oscillator parameters for $\mathrm{Au}$ in Eq. (2) were found in [22, 27] using DESY data. They are as follows: $K=3, \omega_{1}=3.87 \mathrm{eV}$, $f_{1}=59.61(\mathrm{eV})^{2}, g_{1}=2.62 \mathrm{eV}, \omega_{2}=8.37 \mathrm{eV}, f_{2}=122.55(\mathrm{eV})^{2}, g_{2}=6.41 \mathrm{eV}$, $\omega_{3}=23.46 \mathrm{eV}, f_{3}=1031.19(\mathrm{eV})^{2}, g_{3}=27.57 \mathrm{eV}$. Computations were performed at the laboratory temperature $T=300 \mathrm{~K}$ at different experimental separations $a$ with $\omega_{p}=9.0 \mathrm{eV}$ [8, 17]. The obtained magnitudes of the Casimir force are presented in Table 1 (column 2). For comparison in column 3 we present the force magnitudes obtained using the usual plasma model of Eq. (1) with $A(\omega)=0$. Column 4 lists the force magnitudes from the zero-temperature Lifshitz formula using the tabulated optical data for the complex index of refraction (recall that in [16, 17] the experimental data were compared with theory at zero temperature). As is seen from Table 1, at short

Table 1. Magnitudes of the Casimir force at different separations in column 1 computed at $T=300 \mathrm{~K}$ using the generalized plasma-like model (column 2), the usual plasma model (column 3), and at zero temperature using the tabulated optical data for the complex index of refraction (column 4).

\begin{tabular}{cccc}
\hline & \multicolumn{3}{c}{ Force magnitude $(\mathrm{pN})$} \\
\cline { 2 - 4 }$a$ & Generalized & Plasma & Force at zero \\
$(\mathrm{nm})$ & plasma model & model & temperature \\
\hline 60 & 531.1 & 483.2 & 527.4 \\
70 & 358.8 & 332.2 & 356.1 \\
80 & 254.9 & 239.1 & 252.8 \\
90 & 188.2 & 178.3 & 186.5 \\
100 & 143.3 & 136.8 & 141.9 \\
120 & 88.94 & 86.00 & 88.01 \\
150 & 49.30 & 48.19 & 48.71 \\
200 & 22.75 & 22.46 & 22.44 \\
250 & 12.37 & 12.28 & 12.19 \\
300 & 7.478 & 7.438 & 7.355 \\
\hline
\end{tabular}




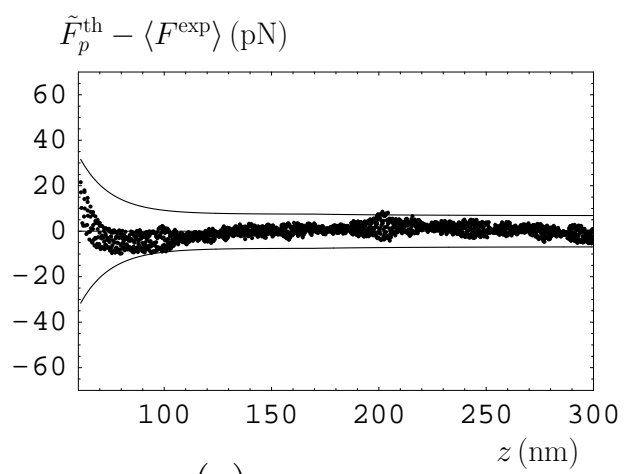

(a)

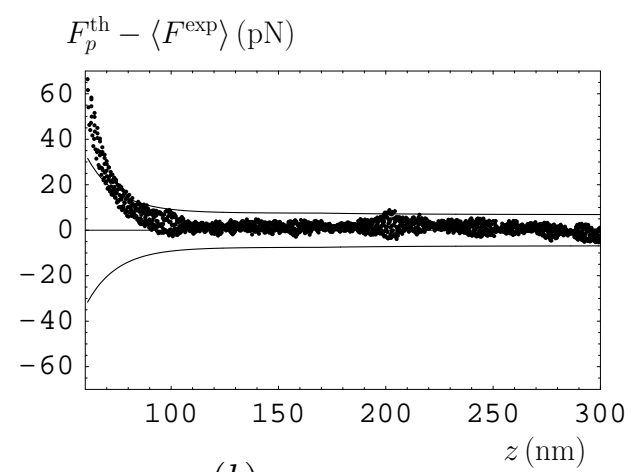

(b)

Figure 2. Differences between the theoretical and mean experimental [16] Casimir forces versus separation. Theoretical forces are computed using $(a)$ the generalized plasma-like model and $(b)$ the usual plasma model.

separations the results from the usual plasma model (column 3) deviate significantly from the predictions of the generalized plasma model (column 2). At the same time the results in column 2 are in close agreement with computations at $T=0$ (column 4 ) which are both consistent with experiment.

Direct comparison with the experimental data of [16] confirms that the generalized plasma model (11), (2) is consistent with measurements of the Casimir force at short separations. In Fig. 2(a) we plot the differences between the theoretical forces computed using the generalized plasma model, $\tilde{F}_{p}^{\text {th }}$, and the mean values of the measured forces. As is seen in Fig. 2(a), almost all points are inside the error bars for the difference between theory and experiment [12, 28] computed at a 95\% confidence level (solid lines). Notice that the comparison of data with the zero-temperature theoretical force practically coincides with that shown in Fig. 2(a). By contrast in Fig. 2(b) the theoretical force, $F_{p}^{\text {th }}$, is computed using the usual plasma model. It is clearly seen that at short separations the usual plasma model is inconsistent with data whereas at larger separations both models are in agreement with measurements.

Refering back to the Introduction, the usual plasma model is consistent with the data of experiment [12] which is the most precise experiment at separations from 160 to $750 \mathrm{~nm}$. At such large separations the predictions of the generalized and usual plasma models almost coincide (in fact, the use of the generalized plasma model instead of the usual one leads to slightly better agreement between experiment and theory in [12). Thus we can conclude that the generalized plasma model (1), (2) both exactly satisfies the Kramers-Kronig relations and is also the only model consistent with all precise experiments on the Casimir force performed to date.

\section{Discussion}

As was mentioned in the Introduction, the usual plasma model neglects the role of free electron scattering. In the absence of scattering the conductivity of free electrons is 
purely imaginary and the dielectric permittivity is completely real signifying the absence of dissipation. The generalized plasma-like dielectric permittivity (11), (2), as well as the usual plasma model, does not take into account the scattering processes of the free electrons. However, it includes dissipation due to the interband transitions of core electrons. These transitions are described by a set of oscillators with nonzero resonant frequencies. Our results demonstrate that the generalized plasma model exactly satisfies the Kramers-Kronig relations and is also consistent with all available experimental data. In the same way, as in [11, 29, 30], it can also be shown that this model is in agreement with the Nernst heat theorem.

The dissipation processes of free electrons on phonons, impurities etc. are not included in the generalized plasma model. They can be taken into account by modifying Eq. (11) to the form

$$
\varepsilon(\omega)-1=A(\omega)-\frac{f_{0}}{\omega\left(\omega+i g_{0}\right)},
$$

where $f_{0}=\omega_{p}^{2}$. The second term on the right-hand side of Eq. (18) is the contribution of an oscillator with zero resonant frequency, $\omega_{0}=0$, which was not included in Eq. (2). This additional oscillator results in a first order pole and leads to the Drude-type term in the dielectric permittivity. The Kramers-Kronig relations for the dielectric permittivity (18) are familiar [2]. However, as was discussed in the Introduction, the use of the dielectric permittivity (18) leads to a violation of the Nernst heat theorem for perfect crystal lattices and to a contradiction with experiment [12. The question why the inclusion of one type of dissipation (interband transitions of core electrons) in the Lifshitz theory is necessary while that of another (scattering processes of free electrons) leads to contradictions with fundamental physical principles and experiment remains open. Future theoretical and experimental developments will shed light on this issue.

\section{Acknowledgments}

This work was supported by the DOE Grant No. DE-FG02-04ER46131. Numerical computations were supported by the NSF Grant No. PHY0355092.

\section{References}

[1] Lifshitz E M and Pitaevskii L P 1980 Statistical Physics Part II (Oxford: Pergamon Press)

[2] Landau L D, Lifshitz E M and Pitaevskii L P 1984 Electrodynamics of Continuous Media (Oxford: Pergamon Press)

[3] Milonni P W 1994 The Quantum Vacuum (San Diego: Academic Press)

[4] Kardar M and Golestanian R 1999 Rev. Mod. Phys. 711233

[5] Bordag M, Mohideen U and Mostepanenko V M 2001 Phys. Rep. 3531

[6] Elizalde E and Odintsov S D (eds) 2006 Papers presented at the 7th Workshop on Quantum Field Theory Under the Influence of External Conditions, J. Phys. A: Mat. Gen. 396109

[7] Casimir H B G 1948 Proc. K. Ned. Akad. Wet. 51793

[8] Palik E D (ed.) 1985 Handbook of Optical Constants of Solids (New York: Academic Press)

[9] Boström M and Sernelius B E 2000 Phys. Rev. Lett. 844757 
[10] Brevik I, Aarseth J B, Høye J S and Milton K A 2005 Phys. Rev. E 71056101

[11] Bezerra V B, Klimchitskaya G L, Mostepanenko V M and Romero C 2004 Phys. Rev. A 69022119

[12] Decca R S, López D, Fischbach E, Klimchitskaya G L, Krause D E and Mostepanenko V M 2005 Ann. Phys. NY 31837

[13] Geyer B, Klimchitskaya G L and Mostepanenko V M 2003 Phys. Rev. A 67062102

[14] Genet C, Lambrecht A and Reynaud S 2000 Phys. Rev. A 62012110

[15] Bordag M, Geyer B, Klimchitskaya G L and Mostepanenko V M 2000 Phys. Rev. Lett. 85503

[16] Harris B W, Chen F and Mohideen U 2000 Phys. Rev. A 62052109

[17] Chen F, Klimchitskaya G L, Mohideen U and Mostepanenko V M 2004 Phys. Rev. A 69022117

[18] Bezerra V B, Decca R S, Fischbach E, Geyer B, Klimchitskaya G L, Krause D E, López D, Mostepanenko V M and Romero C 2006 Phys. Rev. E 730281101

[19] Mostepanenko V M, Bezerra V B, Decca R S, Fischbach E, Geyer B, Klimchitskaya G L, Krause D E, López D and Romero C 2006 J. Phys. A: Mat. Gen. 396589

[20] Høye J S, Brevik I, Aarseth J B and Milton K A 2006 J. Phys. A: Mat. Gen. 396031

[21] Jackson J D 1999 Classical Electrodynamics (New York: John Willey \& Sons)

[22] Parsegian V A 2005 Van der Waals forces: A Handbook for Biologists, Chemists, Engineers, and Physicists (Cambridge: Cambridge University Press)

[23] Kittel C 1976 Introduction to Solid State Physics (New York: John Willey \& Sons)

[24] Emig T, Jaffe R L, Kardar M and Scardicchio A 2006 Phys. Rev. Lett. 96080403

[25] Bordag M 2006 Phys. Rev. D 73125018

[26] Gies H and Klingmüller K 2006 Phys. Rev. Lett. 96220401

[27] Parsegian V A and Weiss G H 1981 J. Colloid. Interface Sci. 81285

[28] Klimchitskaya G L, Chen F, Decca R S, Fischbach E, Krause D E, López D, Mohideen U and Mostepanenko V M 2006 J. Phys. A.: Mat. Gen. 396485

[29] Geyer B, Klimchitskaya G L and Mostepanenko V M 2005 Phys. Rev. D 72085009

[30] Klimchitskaya G L, Geyer B and Mostepanenko V M 2006 J. Phys. A.: Mat. Gen. 396495 